\title{
Implementation of Clinical Video Telemedicine (CVT) within a VA Medical Center Is Cost Effective and Well Received by Veterans
}

\author{
John Wennergren'1, Imtiaz Munshi², Alyssa D. Fajardo' ${ }^{1}$, Virgilio V. George ${ }^{2}$ \\ ${ }^{1}$ Department of Surgery, Indiana University School of Medicine, Indianapolis, USA \\ ${ }^{2}$ Richard Roudebush VA Medical Center, Indianapolis, USA \\ Email: vigeorge@iupui.edu
}

Received 13 April 2014; revised 12 May 2014; accepted 11 June 2014

Copyright (C) 2014 by authors and Scientific Research Publishing Inc.

This work is licensed under the Creative Commons Attribution International License (CC BY).

http://creativecommons.org/licenses/by/4.0/

(c) (i) Open Access

\section{Abstract}

Background: The Veterans Administration (VA) has been using telehealth to enhance Veteran access to high quality VA care for over a decade. Clinical video telehealth (CVT) is one such telehealth tool that allows Veterans the opportunity to be evaluated by specialists at the Indianapolis VA while they actually remain in their community (in their local healthcare setting). Such tools are reported to improve satisfaction by avoiding the need to make the long, stressful, and often costly trips to the Medical Center. Our goal is to describe the results of CVT implementation at the Indianapolis VA. Methods: A retrospective review of the data from 2011-2014 related to the use of CVT at the Indianapolis VA was undertaken. The data collected during this time period included: the number of CVT visits per year by specialty, the number of miles in travel avoided per visit, and patient satisfaction survey data, which are obtained after each CVT visit. Results: A total of 14,708 Veterans have enrolled in our CVT telehealth program since 2011. There were 23,267 visits in 2013. 486,170 miles related to travel were avoided (calculating the number of miles avoided in travel from home to a local satellite site as compared to having to travel from home to the Indianapolis VA). At the current Government reimbursement rate of $\$ 0.42 / \mathbf{m i l e}$, this is expressed in a cost avoidance of $\$ 209,053$. In total, since 2011 , the telehealth CVT program has saved the Government \$331,132, a total of $\mathbf{7 7 0 , 0 7 5}$ miles saved in travel for our Veterans. In addition, the CVT program has been very well received by our Veterans with an overall satisfaction score of $96 \%$. Conclusion: Our results indicate that the implementation of CVT is cost effective and is well received by Veterans. Telehealth modalities such as CVT are viable options that enhance Veteran satisfaction by decreasing the time and the costs related to travel while continuing to offer high quality health care. 


\section{Keywords}

\section{Clinical Video Telemedicine}

\section{Introduction}

The demand for high quality and safe health care in the United States continues to grow and the costs associated with providing this are growing at an even faster and more alarming rate. According to the Centers for Medicare and Medicaid Service (CMS) in 2012 health care spending accounted for approximately 17\% of the GDP (or $\$ 2.8$ trillion) and it is predicted that by 2022 this will increase to $19.9 \%$ of the GDP or $\$ 5$ trillion (a $78 \%$ increase) [1]. The Veteran's Health Administration (VHA) is no exception as it also experiences the pressures related to increased costs paired with increased demand. Recently, the National Center for Veterans Analysis and Statistics (NCVAS) created a Veteran Population Projection Model known as VetPop2011, with projections made out to the year 2040. Although it's projected that the current armed forces numbering just under 23 million will decrease to just over 14 million [2], the cost to provide health care which has increased from $\$ 20$ billion in the year 2000 to around $\$ 35$ billion in 2009 is expected to continue to increase as revenues fall [3]. It is projected, that by the year 2020,52\% of the nation's population over the age of 65 will consist of Veterans, up from $33 \%$ in the year 2000 [4]. With these facts in mind, various groups and organizations have started to reconsider how they provide healthcare services and some even have decided to reduce or eliminate coverage. The VA has opted to implement existing technologies in an attempt to manage the growing healthcare needs of Veterans. A national home telehealth program is one such program where patients with chronic diseases within the VA system are enrolled and receive a number of telecommunication or telehealth devices for in-home use. These devices help physicians monitor the health of their patients directly from the comfort of the patients' home [5]. Such healthcare technology applications have been shown in studies to not negatively impact patient outcomes or quality with regards to morbidity and mortality while allowing for appropriate clinical assessments to be made at lower costs [6] [7]. The use of telehealth technologies is not limited to in home use, but has also been applied in other fields of medicine both at home and within the hospital [8] [9].

In 2011, The Richard L. Roudebush VA Medical Center launched an initiative to implement telehealth for use in clinical management. We believed that this was an exciting new opportunity that would enhance access to care while supporting and maintaining quality. Of the several telehealth tools available for use, clinical video telehealth (CVT) seemed best suited for use in many of our practices. CVT allows the Veterans an opportunity to visit their provider via teleconference from a location close to the patient's home. We know of no published reports with regards to this new and evolving technology as applied to a clinical practice within a VA medical center. Our report describes how we applied CVT in clinical practice and its impact on cost avoidance and on improving patient satisfaction. We also briefly outline our strategy for the dissemination of and the integration of telehealth technologies to support our other rapidly growing clinical practices.

\section{Methods}

Since 2011, the Richard L. Roudebush VA Medical Center established CVT, a clinical network whose goal was to increase access for Veterans within the VA system without compromising quality of care. To date several clinical services utilize this technology with many more services projected to implement this technology into practice in the near future. General Surgery, Chronic Pain, Internal Medicine, Wound Care and Pre-Assessment Testing are just a few services currently using of this technology.

Telehealth allows providers to conduct follow-up appointments with veterans using telecommunications and telehealth technology. A single unit located at a satellite VA site (such as a clinic) is outfitted with the necessary instrumentation required to perform a complete physical examination; everything from the assessment of the cardio/pulmonary system to wound care evaluation. A physician extender (a Licensed Nurse Practitioner, Registered Nurse or a Nurse Practitioner) is co-located at the satellite site and is physically available with the Veteran. As the consulting provider, located at the Indianapolis VA telehealth suite, conducts his or her clinic visit with the Veteran, the physician extender is asked to perform any of the abovementioned tasks, which may include obtaining a 12-lead electrocardiogram, if necessary. All results including vital signs can be reviewed by 
the provider in real-time. To provide an example, as the physician extender conducts a cardiovascular exam, the provider located at the Indianapolis VA can hear the heart sounds simultaneously. As the physician extender performs other components of the physical examination they can relay information to the provider who can then devise a treatment plan for the Veteran.

From 2011 to 2013 a total of 14,708 Veterans have enrolled to use telehealth. Data related to the number of miles traveled between the Veterans home to a satellite site versus the distance traveled from the Veteran's home to the Roudebush VA Medical Center have been collected. The mileage saved in travel for each Veteran is registered as costs avoided - the amount of money saved by the VA for not having to pay the travel reimbursement costs related to the Veteran driving to the Medical Center. On average, a Veteran is reimbursed 42 cents per mile. This data is presented below (Table 1).

Additionally, at each encounter a CVT satisfaction survey is provided to the veteran at the completion of the visit. This confidential survey consists of 12 questions ranging from how comfortable they felt with the equipment, if they were able to see and hear the provider clearly, if they received good care and if they would use the system again. Veterans were encouraged to mail their survey back to the office once complete. The time to complete the survey was approximately 3 minutes. Responses were rated on a 5 point scale ranging from strongly agree to strongly disagree. Survey results for the year 2013 are presented below (Table 2).

\section{Results}

Data was collected and analyzed from 38 satellite sites that refer patients to the VA Medical Center in Indianapolis. 35 CVT units were purchased at a cost of approximately $\$ 50,000$ per unit (Image 1 ). In the 2013 calendar

\begin{tabular}{|c|c|c|}
\hline \multicolumn{3}{|c|}{ Miles Saved } \\
\hline & Patient Miles Saved & Reduction in Benefit Travel \\
\hline FY11 & 72,628 & $\$ 31,230$ \\
\hline FY12 & 495,182 & $\$ 212,928$ \\
\hline FY13 & 972,340 & $\$ 418,106$ \\
\hline total & $1,540,150$ & $\$ 662,264$ \\
\hline
\end{tabular}

Table 2. Satisfaction data for FY 2013.

\begin{tabular}{cc} 
Question & V11 \\
Technology Expert & 4.8 \\
Video Quality & 4.83 \\
Sound Quality & 4.81 \\
Technical Assistance & 4.82 \\
Provider Relationship & 4.61 \\
Convenient & 4.73 \\
Needs Met & 4.77 \\
Received Good Care & 4.85 \\
Care Expected & 4.8 \\
Overall & 4.79 \\
Recommend & 4.81 \\
Dec Travel & 4.84 \\
Pt Satisfaction Mean Score & 4.79 \\
Satisfaction & $96 \%$ \\
\hline
\end{tabular}


year 23,267 visits were made to local satellite sites using CVT technology. When analyzing the number of miles saved traveling to these sites for routine follow up visits rather than traveling to Indianapolis, a total of 1,540,150 travel miles were avoided. Data for miles saved traveling for 2011 and 2012 fiscal years are also included in Table 1. When calculating travel reimbursements that otherwise would have been paid out to these Veterans since inception of the program, an estimated \$662,264 has been saved.

In addition, an average of 90 miles per Veteran is avoided in traveling. This in turn saves the Veteran approximately one and a half hours of travel time on average. Upon completion of their visit, Veterans were asked to fill out a confidential survey consisting of 12 questions (Figure 1). For the year 2013, answers to all questions were scored at either a 4 or 5 level with a satisfaction score of $96 \%$. The response rate was $58 \%$.

\section{Discussion}

The largest integrated healthcare system in the United States is the Veterans Administration (VA). The VA delivers healthcare to more than 6 million unique patients using a variety of traditional and now more innovative clinical practices. Such innovations include the implementation of and the use of telemedicine or telehealth. One of Secretary of Veterans Affairs Eric Shinseki's goals has been to transform the VA into “ $\ldots$ a $21^{\text {st }}$ century organization that is people-centric, results driven and forward thinking”. The major goals associated with use of technology have been to increase access, improve efficiency and enhance veteran satisfaction.

Telehealth based care was initially implemented as a three year pilot program in 2000 for 800 Veterans and when determined to be successful it was fully promoted beginning in 2003 as a viable option for the delivery of high quality health care to Veterans. It has now been fully integrated into almost all aspects of care as an essential component within the VA health care system. Secretary Shinseki noted in his Performance and Accountability Report that in 2012 the VA had provided care to 500,000 Veterans using 1.4 million telehealth-based consultations delivered from 150 VA Medical Centers and 750 Outpatient Clinics. Of those, 148,000 Veterans participated in home-based consultations, 119,000 received telehealth care directly within their homes, and 76,000 received telemental health consultations. Implementation of these types of services clearly expands Veteran access to high quality primary care and specialty care. This type of care coordination has resulted in a 58\% decrease in hospital bed days and a 38\% reduction in admissions. The VA is ahead of many private institutions and health care organizations with respect to the successful implementation of innovations such as, an electronic

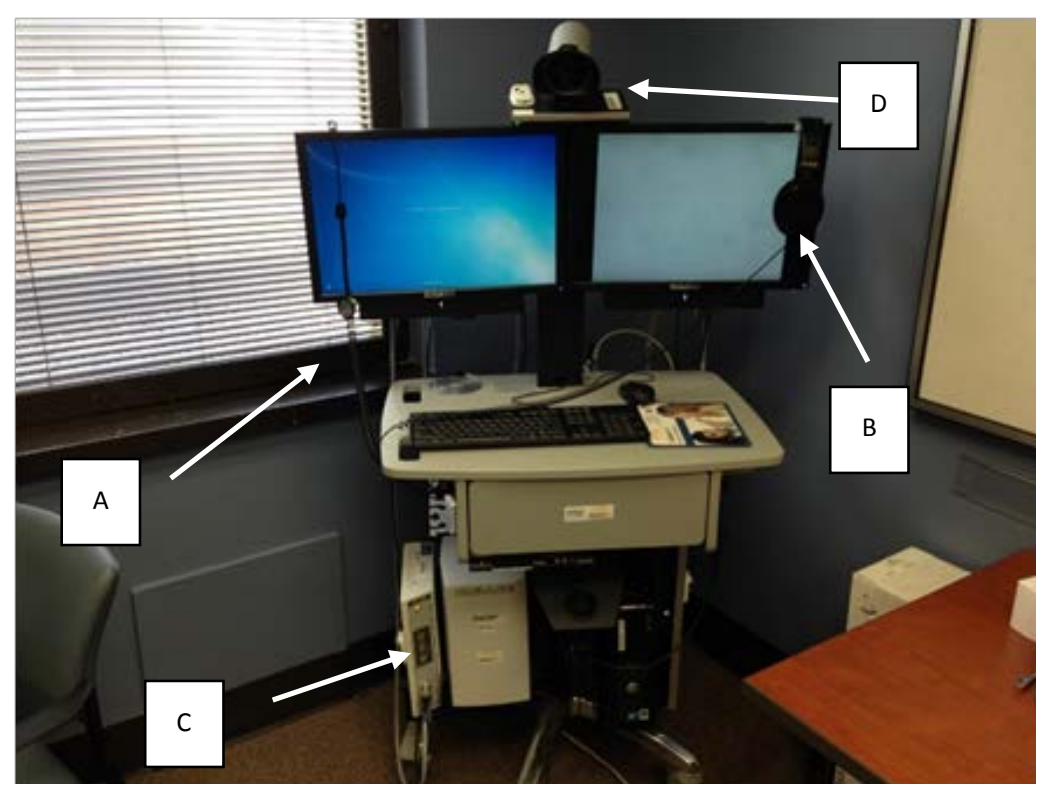

Figure 1. This is a representation of a complete unit (the Iron Bow) provided by GlobalMed Telemedicine. (A) Electronic stethoscope; (B) High definition stereo headphones; (C) Accessory for additional instrumentation such as 12 lead EKG, high definition cameras to evaluate wound care, otoscope, etc.; (D) High definition camera. 
health record system and the wide dissemination and integration of telehealth in clinical practice. It is also the leader in the successful integration of telehealth data into an electronic health record.

The VA's mission is to provide our Veterans with the "right care in the right place at the right time" this is effectively supported through the appropriate use of telehealth technologies. The goal is to make the home or the local community center the safe and desired place of care when appropriate. The implications of this is quite significant: 1) It means not having to travel to the Medical Center thus, reducing or even eliminating travel time and the anxiety associated with traveling or with missing work; 2) Reducing or eliminating the costs associated with travel (as the VA reimburses Veterans for travel) and avoiding the stress associated with parking; 3) minimizing missed opportunities, no-show rates, and improving access by increasing clinic capacity enabling providers to schedule more new patients; 4) Medical Centers realize a potential cost avoidance when not using existing clinic space, resources or staffing. Overall, the most important impact of telehealth is on Veteran satisfaction - as providing medical services near the home or in the homes increases Veteran satisfaction considerably. Beyond the home and clinic setting, as Secretary Shinseki revealed, the use of apps on smartphones and tablets to treat mental illness such as PTSD are currently in use and evolving to apply this new and exciting technology "on the go" [10].

The three major types of telehealth techniques currently used by VA providers include: clinical video telehealth (CVT), home telehealth (HT) and care coordination store and forward (CCSF). CVT offers VA clinicians synchronous real time, live, secure, and reliable video conferencing. It allows them to safely and accurately assess a patient to then define strategies for management without being in the same location. The Indianapolis VA medical center has implemented CVT quite effectively in the following clinical areas: Mental Health, Nutrition and Dietary, Spinal Cord Injury, Dermatology, MOVE program (weight management), Chaplain Services, Marriage Counseling, Anger Management, Smoking Cessation, Outpatient Substance Abuse programs, Genetic Counseling, Interventional Pain, pre and post-surgical clinical services, and Pharmacy. In 2011, we introduced the use of CVT to the surgical providers in the specialties of colorectal surgery and general surgery. Equipment and training was provided by the Telehealth staff and outcomes measured using a satisfaction survey and determining the costs avoided by eliminating travel pay. In the first year of the pilot (fiscal year 2011) CVT was used twice, by fiscal year 2012 CVT was used to perform 178 consults and visits, and in the first month of fiscal year 2013 we have already used it 26 times to evaluate Veterans; on track to evaluate over 260 Veterans using CVT. We have demonstrated that Surgeons can quickly learn to use CVT and that they can effectively use it to evaluate and assess patients. Patient satisfaction was improved and cost related to travel reimbursement was reduced significantly.

Although it is projected that the number of active armed service members will decrease by the year 2040, those veterans over the age of 65 will continue to increase as will the cost to provide health care [2] [3]. In addition, it is projected that the number of primary care physicians will fall by 91,000 over the next 10 years leading to decreased access to care [11]. Telemedicine is an evolving technology pioneered to address these projections by providing improved access to care without compromising quality medical care. A recent report released by an information and analytics firm shows that by the year 2018, the use of telehealth services will increase from its current level of around $\$ 230$ million per year to $\$ 1.9$ billion per year with an increase in the number of patients using this technology to around 3.2 million, up from 250,000 in 2013. This increase is led in part to recent changes enacted by the Affordable Care Act (ACA). With a projected 32 million additional Americans entering the health system and the baby boom generation coming of age and using Medicare services, many are beginning to realize that telemedicine will help to address the problem of providing timely access to healthcare for this subset of the population particularly even more challenging as we are also faced with shortages in primary care providers along with ever increasing costs of providing health care.

As has been shown previously, this technology has already been implemented within the home setting in the treatment of Veterans with chronic health problems such as CHF, DM, COPD, etc. without compromising outcomes and quality. Grabowski and O’Malley in their study showed that the cost related to hospital admissions from nursing homes with their associated complications and morbidity cost our healthcare system more than \$1 billion annually [12]. Part of these unnecessary admissions may in part be due to the unavailability of a physician on site off hours to evaluate patients for transfer and therefore by default are sent to the hospitals. After implementing telehealth services in 6 of 11 nursing homes, they were able to demonstrate a statistically significant reduction in the hospital admission rates between these two groups with an estimated annual cost savings of around $\$ 120,000$ per nursing home [13]. This is again consistent with data from within the VA system. 
Hwa and Wren's study published last year also demonstrated that when applied in the appropriate ambulatory setting, the follow up of patients postoperatively using telemedicine did not result in a higher complication rate [14]. In addition, patients willingly participated and had greater satisfaction with the system when the cost of travel and time for travel were taken into consideration. Looking at this from the aspect of the clinics and hospitals, they benefit by potentially increasing access to see new patients, or by realizing the indirect cost savings in terms of reduced resource and staff utilization [13].

\section{Conclusion}

Review of the telehealth initiative at the Richard L. Roudebush VA Medical Center in Indianapolis shows that CVT is cost effective not only in saving our Veterans time in terms of travel to their follow-up appointment, but also in saving the VA money in terms of eliminating travel pay reimbursements. In addition, veterans are very satisfied with the way in which the technology is being utilized to assist in their overall care. Telehealth technology has been implemented successfully across a broad range of outpatient and in-patient hospital settings. It is a feasible solution that can help tackle the problem we currently face within our country-the rising demand of timely and high quality healthcare while helping to curb the associated costs, address the drop in provider numbers all while continuing to maintain a high level of satisfaction among the veteran population.

\section{References}

[1] (2013) National Health Expenditures Projections Report 2012-2022. Center for Medicare and Medicaid Services.

[2] (2011) Veteran Population Projections (VetPop). Office of Actuary, Department of Veterans Affairs.

[3] (2007) Geographic Distribution of Expenditures Reports, 2000 to 2009; Veteran Population Projection Model (VetPop). Department of Veterans Affairs.

[4] (2008) Population Estimates. US Census Bureau.

[5] Darkins, A., Ryan, P., Kobb, R., Foster, L., Edmonson, E., Wakefield, B. and Lancaster, A.E. (2008) Care Coordination/Home Telehealth: The Systematic Implementation of Health Informatics, Home Telehealth, and Disease Management to Support the Care of Veteran Patients with Chronic Conditions. Telemedicine Journal and E-Health, 14, 1118-1126. http://dx.doi.org/10.1089/tmj.2008.0021

[6] Finkelstein, S.M., Speedie, S.M. and Potthoff, S. (2006) Home Telehealth Improves Clinical Outcomes at Lower Cost for Home Healthcare. Telemedicine Journal and E-Health, 12,128-136. http://dx.doi.org/10.1089/tmj.2006.12.128

[7] Young, L.B., Chan, P.S., Lu, X., Nallamothu, B.K., Sasson, C. and Cram, P.M. (2011) Impact of Telemedicine Intensive Care Unit Coverage on Patient Outcomes: A Systematic Review and Meta-Analysis. Archives of Internal Medicine, 171, 498-506. http://dx.doi.org/10.1001/archinternmed.2011.61

[8] Hopp, F., Woodbridge, P., Subramanian, U., Copeland, L., Smith, D. and Lowery, J. (2006) Outcomes Associated with a Home Care Telehealth Intervention. Telemedicine Journal and E-Health, 12, 297-307. http://dx.doi.org/10.1001/archinternmed.2011.61

[9] Fortney, J.C., Maciejewski, M.L., Tripathi, S.P., Deen, T.L. and Pyne, J.M. (2011) A Budget Impact Analysis of Telemedicine-Based Collaborative Care for Depression. Medical Care, 49, 872-880. http://dx.doi.org/10.1097/MLR.0b013e31821d2b35

[10] (2012) VA Performance and Accountability Report. Secretary of Veterans Affairs, The Honorable Eric K. Shinseki.

[11] Frisch, S. (2013) The Primary Care Physician Shortage. British Medical Journal, 347, f6559. http://dx.doi.org/10.1136/bmj.f6559

[12] Grabowski, D.C. and O’Malley, A.J. (2014) Use of Telemedicine Can Reduce Hospitalizations of Nursing Home Residents and Generate Savings for Medicare. Health Affairs (Millwood), 33, 244-250. http://dx.doi.org/10.1377/hlthaff.2013.0922

[13] Bendixen, R.M., Levy, C.E., Olive, E.S., Kobb, R.F. and Mann, W.C. (2009) Cost Effectiveness of a Telerehabilitation Program to Support Chronically ILL and Disabled Elders in Their Homes. Telemedicine Journal and E-Health, 15, 3138. http://dx.doi.org/10.1089/tmj.2008.0046

[14] Hwa, K. and Wren, S.M. (2013) Telehealth Follow-Up in Lieu of Postoperative Clinic Visit for Ambulatory Surgery: Results of a Pilot Program. JAMA Surgery, 148, 823-827. http://dx.doi.org/10.1001/jamasurg.2013.2672 
Scientific Research Publishing (SCIRP) is one of the largest Open Access journal publishers. It is currently publishing more than 200 open access, online, peer-reviewed journals covering a wide range of academic disciplines. SCIRP serves the worldwide academic communities and contributes to the progress and application of science with its publication.

Other selected journals from SCIRP are listed as below. Submit your manuscript to us via either submit@scirp.org or Online Submission Portal.
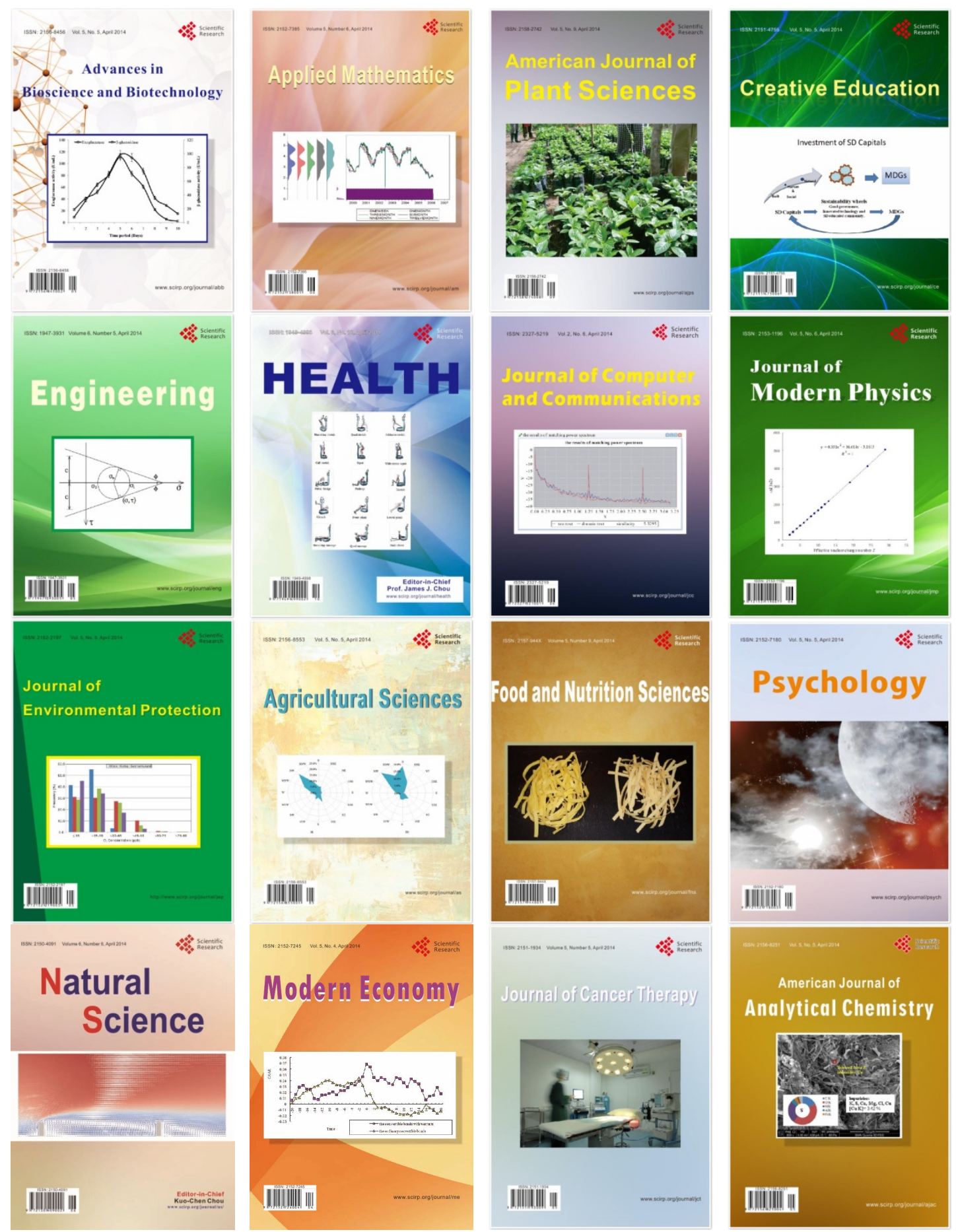